Research Article

\title{
Two Novel Grey System Models and Their Applications on Landslide Forecasting
}

\author{
De-Yi Ma ${ }^{1,2}$ and Jian-Lin $L i^{1,2}$ \\ ${ }^{1}$ Key Laboratory of Geological Hazards on Three Gorges Reservoir Area, China Three Gorges University, Ministry of Education, \\ Yichang 443002, China \\ ${ }^{2}$ College of Hydraulic \& Environmental Engineering, China Three Gorges University, Yichang 443002, China
}

Correspondence should be addressed to De-Yi Ma; mdysave@163.com

Received 30 July 2016; Accepted 25 October 2016

Academic Editor: Enrique Onieva

Copyright (C) 2016 D.-Y. Ma and J.-L. Li. This is an open access article distributed under the Creative Commons Attribution License, which permits unrestricted use, distribution, and reproduction in any medium, provided the original work is properly cited.

For the small sample poor information, grey model is one of the good forecasting models. However, the simulation curve of original data is not consistent with that of the data by translations. In this paper, we present two novel grey system models, that is, generalized grey model and generalized discrete grey model. Compared with grey model, we prove that the simulation curve of original data is consistent with that of the new data by translations for the novel grey model, which was also demonstrated by the results of practical numerical examples.

\section{Introduction}

There are many uncertain problems in our lives. In order to analyze deeply these problems, grey model $(\operatorname{GM}(1,1))$ was proposed by Deng [1]. Many researchers focused on the study of grey model since it was presented. $\operatorname{GM}(1,1)$ has a wide field of application. Based on the characteristic of different data sequence, lots of researchers improve the fitting accuracy of $\operatorname{GM}(1,1)$ from various angles. Besides $\operatorname{GM}(1,1)$, Connotation grey model was also proposed by Deng [2] The relation between Connotation grey model and GM $(1,1)$ was further studied. When development coefficient is small, the above two models are interchangeable. Moreover, the uniform upper bounds of relative error were given by Liu et al. [3].

Later on, $\operatorname{GM}(1,1)$ was extended to discrete grey system $(\operatorname{DGM}(1,1))$ by Xie and Liu [4]. Because DGM $(1,1)$ does not need the discrete approximation, it usually gets small simulation error. After exploring the effect of original data tiny disturbance in depth, Wu et al. [5] proposed the fractional order accumulation grey model which could effectively reduce disturbance of the grey model. Fractional order grey model was proposed by Mao et al. [6] and the corresponding image equation was extended to the fractional order differential equation. $\operatorname{GM}(1,1)$ was extended to partial grey model by Liu $[7,8]$ and the corresponding property was studied. By extreme learning machines, grey model was extended to GrELM by Liu and Fu [9] and solved the volatility forecasting problems of interbank offered rate. $\operatorname{GM}(1,1)$ was also extended to other models in $[10,11]$.

However, for the aforementioned models, it is usually supposed that original data are nonnegative. If the original data contain negative and each number plus a constant, the effect of the simulation forecasting of $\operatorname{GM}(1,1)$ was researched by Li [12]. Feng [13] proved that the different initial data yield different simulation data. Li [14] showed that the original data consisting of negative are suitable for $\operatorname{GM}(1,1)$ through multiply transform.

Observation data, for example, landslide observation data, are usually recorded relative to a reference point. Besides, studying landslide does not need to start with the first data every time. Sometimes the situation after a period of landslide also deserves to be investigated. In this case, we should choose a reference point. However, different reference point has different simulation data of $\operatorname{GM}(1,1)$. This will reduce the reliability and consistency.

In this paper, in order to ensure that the simulation curve of original data is consistent with that of the 
data by translations, we propose generalized grey system $(\operatorname{GGM}(1,1))$ and generalized discrete grey system $(\operatorname{GDGM}(1,1))$. Compared with grey model, we prove that the simulation curve of original data is consistent with that of the data by translations for the novel grey model. At last, example on landslide has further demonstrated that the simulation effects of two novel grey models have no relation with reference point.

The remainder of this paper is organized as follows. In Section 2, the definitions of $\operatorname{GM}(1,1)$ and $\operatorname{DGM}(1,1)$ are introduced. $\operatorname{GGM}(1,1)$ and $\operatorname{GDGM}(1,1)$ are presented and their translation consistence is proved in Sections 3 and 4, respectively. In Section 5, through three examples, we compare the simulation effect of new $\operatorname{GGM}(1,1)$ and $\operatorname{GDGM}(1,1)$ with that of $\operatorname{GM}(1,1)$ and $\operatorname{DGM}(1,1)$, respectively. An empirical analysis of landslide forecasting in BaZiMen area is discussed in Section 6. In the last section we give the conclusions of this paper.

\section{Preliminaries}

For quick later reference we collect some notations and basic facts about grey system. Good general reference for the theory of grey system is the book of Deng [2].

Let the original data sequence be

$$
X^{(0)}=\left\{x^{(0)}(1), x^{(0)}(2), \ldots, x^{(0)}(n)\right\}
$$

where $x^{(0)}(k) \geq 0, k=1,2, \ldots, n$. Then the first order accumulated sequence of the original data sequence is

$$
X^{(1)}=\left\{x^{(1)}(1), x^{(1)}(2), \ldots, x^{(1)}(n)\right\}
$$

where $x^{(1)}(k)=\sum_{i=1}^{k} x^{(0)}(i), k=1,2, \ldots, n$.

As a consequence, the mean generated sequence of the first order accumulated sequence could be expressed as

$$
Z^{(1)}=\left\{z^{(1)}(2), z^{(1)}(3), \ldots, z^{(1)}(n)\right\},
$$

where $z^{(1)}(k)=(1 / 2)\left(x^{(1)}(k)+x^{(1)}(k-1)\right), k=2,3, \ldots, n$.

Based on the above notations, we introduce $\operatorname{GM}(1,1)$ and its character as follows.

Definition 1 (see [15]). $x^{(0)}(k)+a z^{(1)}(k)=b$ is called mean form of $\operatorname{GM}(1,1)$.

Definition 2 (see [15]). $d x^{(1)} / d t+a x^{(1)}=b$ is called Whitenization equation of $\operatorname{GM}(1,1)$.

By the definition of the grey model, we could easily derive the following results.

Lemma 3 (see [15]). The following equation

$$
\widehat{x}^{(1)}(k)=\left(x^{(0)}(1)-\frac{b}{a}\right) e^{-a(k-1)}+\frac{b}{a}, \quad k=1,2, \ldots, n
$$

is called the time response equation of $\operatorname{GGM}(1,1)$.
Lemma 4 (see [15]). Let $\widehat{\beta}=(a, b)^{T}$ be a sequence of parameters. Denote

$$
\begin{aligned}
& Y=\left(\begin{array}{c}
x^{(1)}(2) \\
x^{(1)}(3) \\
\vdots \\
x^{(1)}(n)
\end{array}\right), \\
& B=\left(\begin{array}{cc}
-z^{(1)}(2) & 1 \\
-z^{(1)}(3) & 1 \\
\vdots & \vdots \\
-z^{(1)}(n) & 1
\end{array}\right) .
\end{aligned}
$$

Then the least squares estimate parameter of $G M(1,1)$ equation

$$
x^{(0)}(k)+a z^{(1)}(k)=b
$$

satisfies $\widehat{\beta}=\left(B^{T} B\right)^{-1} B^{T} Y$.

In what follows, we introduce the definition of $\operatorname{DGM}(1,1)$ and its characters.

Definition 5 (see [4]). $x^{(1)}(k+1)=\beta_{1} x^{(1)}(k)+\beta_{2}$ is called $\operatorname{DGM}(1,1)$.

Lemma 6 (see [4]). The following equation

$$
\begin{aligned}
\hat{x}^{(1)}(k)=\left(x^{(0)}(1)-\frac{\beta_{2}}{1-\beta_{1}}\right) \beta_{1}^{k}+\frac{\beta_{2}}{1-\beta_{1}} & \\
& k=1,2, \ldots, n
\end{aligned}
$$

is called the time response equation of $\operatorname{DGM}(1,1)$.

Lemma 7 (see [4]). If $\widehat{\beta}=\left(\beta_{1}, \beta_{2}\right)^{T}$ is a sequence of parameters and

$$
\begin{aligned}
& Y=\left(\begin{array}{c}
x^{(1)}(2) \\
x^{(1)}(3) \\
\vdots \\
x^{(1)}(n)
\end{array}\right), \\
& B=\left(\begin{array}{cc}
x^{(1)}(1) & 1 \\
x^{(1)}(2) & 1 \\
\vdots & \vdots \\
x^{(1)}(n-1) & 1
\end{array}\right),
\end{aligned}
$$

then the least squares estimate parameter of $D G M(1,1)$ equation

$$
x^{(1)}(k+1)=\beta_{1} x^{(1)}(k)+\beta_{2}
$$

satisfies $\widehat{\beta}=\left(B^{T} B\right)^{-1} B^{T} Y$. 


\section{The Proposed Generalized Grey Model $(\operatorname{GGM}(\mathbf{1}, \mathbf{1}))$}

In this section, we are going to investigate the proposed forecasting model $\operatorname{GGM}(1,1)$. We first give the definition of $\operatorname{GGM}(1,1)$ and then derive its time response equation.

Assume $h \in R$. Let the original data sequence be

$$
X^{*(0)}=\left\{x^{*(0)}(1), x^{*(0)}(2), \ldots, x^{*(0)}(n)\right\},
$$

where $x^{*(0)}(k)=x^{(0)}(k)+h, k=1,2, \ldots, n$. Then we have the first order accumulated sequence of the original data as follows:

$$
X^{*(1)}=\left\{x^{*(1)}(1), x^{*(1)}(2), \ldots, x^{*(1)}(n)\right\},
$$

where $x^{*(1)}(k)=\sum_{i=1}^{k} x^{*(0)}(i), k=1,2, \ldots, n$. As a result, the mean generated sequence of the first order accumulated sequence writes as

$$
Z^{*(1)}=\left\{z^{*(1)}(2), z^{*(1)}(3), \ldots, z^{*(1)}(n)\right\},
$$

where $z^{*(1)}(k)=(1 / 2)\left(x^{*(1)}(k)+x^{*(1)}(k-1)\right), k=2, \ldots, n$.

Given the above notations, we now present the definition of $\operatorname{GGM}(1,1)$.

Definition 8. The following equation

$$
x^{*(0)}(k)+a^{*} z^{*(1)}(k)=b^{*}+h+a^{*} h(k-0.5)
$$

is called mean form of $\operatorname{GGM}(1,1)$.

Definition 9. Whitenization differential equation of GGM(1, $1)$ is

$$
\frac{d x^{*(1)}}{d t}+a x^{*(1)}=b^{*}+h+a^{*} h t .
$$

Based on the above definitions, we could derive the following.

Theorem 10. The following equation,

$$
\begin{array}{r}
\hat{x}^{*(1)}(k)=\frac{b^{*}}{a^{*}}+\left(x^{*(1)}(1)-h-\frac{b^{*}}{a^{*}}\right) e^{-a^{*}(k-1)}+h k, \\
k=1,2, \ldots, n,
\end{array}
$$

is called the time response equation of $\operatorname{GGM}(1,1)$.

Proof. The solution of differential equation $d x^{*(1)} / d t+$ $a x^{*(1)}=b^{*}+h+a^{*} h t$ is $\widehat{x}^{*(1)}(t)=b^{*} / a^{*}+c e^{-a^{*} t}+h t$. If initial datum satisfies $\hat{x}^{*(1)}(1)=x^{*(1)}(1)$, then $\hat{x}^{*(1)}(t)=$ $b^{*} / a^{*}+\left(x^{*(1)}(1)-h-b^{*} / a^{*}\right) e^{-a^{*}(t-1)}+h t$.

It follows that

$$
\begin{array}{r}
\hat{x}^{*(1)}(k)=\frac{b^{*}}{a^{*}}+\left(x^{*(1)}(1)-h-\frac{b^{*}}{a^{*}}\right) e^{-a^{*}(k-1)}+h k, \\
k=1,2, \ldots, n .
\end{array}
$$

This finishes the proof of Theorem 10 .
Theorem 11. If $x^{(0)}(k) \geq 0, k=1,2, \ldots, n$, and $h \geq 0$, then $\operatorname{GGM}(1,1)$ is reduced to $\operatorname{GM}(1,1)$.

In other words, If $x^{*(0)}(k)=x^{(0)}(k)+h, k=1,2, \ldots, n$, and $x^{(0)}(k) \geq 0, k=1,2, \ldots, n, h \geq 0$, then $a^{*}=a, b^{*}=b$, and $\widehat{X}^{*(0)}=\widehat{X}^{(0)}+h$.

Proof. If $x^{*(0)}(k)=x^{(0)}(k)+h, k=1,2, \ldots, n$, then $x^{*(1)}(k)=$ $x^{(1)}(k)+k h, k=1,2, \ldots, n$.

In view of (12), we have

$$
\begin{aligned}
z^{*(1)}(k) & =0.5\left(x^{*(1)}(k)+x^{*(1)}(k-1)\right) \\
& =0.5\left(x^{(1)}(k)+x^{(1)}(k-1)\right)+(k-0.5) h \\
& =z^{(1)}(k)+(k-0.5) h, \quad k=2,3, \ldots, n .
\end{aligned}
$$

Plugging (17) into (13), we arrive at

$$
\begin{aligned}
x^{(0)} & (k)+h+a^{*} z^{(1)}(k)+a^{*} h(k-0.5) \\
= & b^{*}+h+a^{*} h(k-0.5) .
\end{aligned}
$$

Therefore,

$$
x^{(0)}(k)+a^{*} z^{(1)}(k)=b^{*} .
$$

This equation is the same as the mean form of $\operatorname{GM}(1,1)$. That is, $a^{*}=a$ and $b^{*}=b$. By Theorem 10 , one gets

$$
\hat{x}^{*(1)}(k)=\frac{b^{*}}{a^{*}}+\left(x^{*(1)}(1)-h-\frac{b^{*}}{a^{*}}\right) e^{-a^{*}(k-1)}
$$

$+h k$,

$$
\begin{aligned}
a^{*} & =a, \\
b^{*} & =b, \\
x^{*(1)}(1) & =x^{(1)}(1)+h, \\
\text { so } \hat{x}^{*(1)}(k) & =\widehat{x}^{(1)}(k)+h k .
\end{aligned}
$$

Then

$$
\begin{aligned}
\hat{x}^{*(0)}(k) & =\widehat{x}^{(1)}(k)-\widehat{x}^{*(1)}(k-1) \\
& =\widehat{x}^{(1)}(k)+h k-\widehat{x}^{(1)}(k-1)-h(k-1) \\
& =\widehat{x}^{(0)}(k)+h .
\end{aligned}
$$

Therefore, $\widehat{X}^{*(0)}=\widehat{X}^{(0)}+h$ and this finishes the proof of Theorem 11.

Remark 12. By Theorem 11, it is clear that the simulation curve of $\operatorname{GGM}(1,1)$ is consistent with that of $\operatorname{GM}(1,1)$ for the nonnegative original data.

\section{The Proposed Generalized Discrete Grey Model (GDGM(1, 1))}

In this section, we will investigate the proposed forecasting model $\operatorname{GDGM}(1,1)$. We first give the definition of $\operatorname{GDGM}(1,1)$ and then derive its time response equation. 
Definition 13. The following equation,

$$
x^{*(1)}(k+1)=\beta_{1}^{*} x^{*(1)}(k)+\beta_{2}^{*}-\beta_{1}^{*} k h+(k+1) h,
$$

is called mean form of $\operatorname{GDGM}(1,1)$.

The following theorem is provided to discuss the time response equation of $\operatorname{GDGM}(1,1)$.

Theorem 14. The following equation,

$$
\begin{aligned}
\hat{x}^{*(1)}(k+1)= & \beta_{1}^{* k} x^{*(1)}(1)+\frac{1-\beta_{1}^{* k}}{1-\beta_{1}^{*}}\left(h+\beta_{2}^{*}\right) \\
- & \beta_{1}^{* k} h+(k+1) h, \\
& \quad k=1,2, \ldots, n-1,
\end{aligned}
$$

is called the time response equation of $\operatorname{GDGM}(1,1)$.

Proof.

$$
\begin{array}{r}
\widehat{x}^{*(1)}(k+1)=\beta_{1}^{*} \widehat{x}^{*(1)}(k)+\beta_{2}^{*}-\beta_{1}^{*} k h+(k+1) h \\
=\beta_{1}^{*}\left[\widehat{x}^{*(1)}(k-1)+\beta_{2}^{*}-\beta_{1}^{*}(k-1) h+k h\right]+\beta_{2}^{*} \\
-\beta_{1}^{*} k h+(k+1) h=\cdots \\
=\beta_{1}^{* k} x^{*(1)}(1)+\frac{1-\beta_{1}^{* k}}{1-\beta_{1}^{*}} \beta_{2}^{*}-\beta_{1}^{*} h+(k+1) h, \\
k=1,2, \ldots, n-1 .
\end{array}
$$

This proves Theorem 14 .

To obtain the relation between $\operatorname{GDGM}(1,1)$ and $\operatorname{DGM}(1,1)$, we have the following.

Theorem 15. If $x^{(0)}(k) \geq 0, k=1,2, \ldots, n$, and $h \geq 0$, then $\operatorname{GDGM}(1,1)$ is reduced to $\operatorname{DGM}(1,1)$.

In other words, provided $x^{*(0)}(k)=x^{(0)}(k)+h, k=$ $1,2, \ldots, n$, and $x^{(0)}(k) \geq 0, k=1,2, \ldots, n, h \geq 0$, then

$$
\begin{aligned}
\beta_{1}^{*} & =\beta_{1}, \\
\beta_{2}^{*} & =\beta_{2}, \\
\hat{x}^{*(0)}(k) & =\hat{x}^{(0)}(k)+h .
\end{aligned}
$$

Proof. The equation of $\operatorname{GDGM}(1,1)$ is $x^{*(1)}(k+1)=$ $\beta_{1}^{*} x^{*(1)}(k)+\beta_{2}^{*}-\beta_{1}^{*} k h+(k+1) h$, and $x^{*(1)}(k)=x^{(1)}(k)+k h$; then,

$$
\begin{aligned}
x^{(1)} & (k+1)+(k+1) h \\
& =\beta_{1}^{*}\left(x^{(1)}(k)+k h\right)+\beta_{2}^{*}-\beta_{1}^{*} k h+(k+1) h .
\end{aligned}
$$

Thus $x^{(1)}(k+1)=\beta_{1}^{*} x^{(1)}(k)+\beta_{2}^{*}$, which implies $\beta_{1}^{*}=\beta_{1}$, $\beta_{2}^{*}=\beta_{2}$.
By Theorem 14, that is, $\widehat{x}^{*(1)}(k+1)=\beta_{1}^{* k} x^{*(1)}(1)+((1-$ $\left.\left.\beta_{1}^{* k}\right) /\left(1-\beta_{1}^{*}\right)\right)\left(h+\beta_{2}^{*}\right)-\beta_{1}^{* k} h+(k+1) h$, and $x^{*(1)}(1)=x^{(1)}(1)+$ $h$, we have

$$
\begin{aligned}
\hat{x}^{*(1)}(k+1)= & \beta_{1}^{k} x^{(1)}(1)+\frac{1-\beta_{1}^{k}}{1-\beta_{1}}\left(h+\beta_{2}\right) \\
& +(k+1) h=\hat{x}^{(1)}(k+1)+(k+1) h,
\end{aligned}
$$

and therefore $\widehat{x}^{*(0)}(k)=\widehat{x}^{(0)}(k)+h$. We complete the proof of Theorem 15.

Remark 16. From Theorem 15, it is clear that the simulation curve of $\operatorname{GDGM}(1,1)$ is consistent with $\operatorname{DGM}(1,1)$ for the nonnegative original data.

\section{Verification of $\operatorname{GGM}(1,1)$ and $\operatorname{GDGM}(1,1)$}

The simulation effects of $\operatorname{GGM}(1,1)$ and $\operatorname{GDGM}(1,1)$ are evaluated via the following three real cases in this section.

Case 1 (the example for numeric data). Li [16] used the numeric data to discuss the conflict between desire for a good smoothing effect and desire to give additional weight to the recent change, but the author does not give a method for solving such a conflict. Wu et al. [17] presented $\operatorname{GDES}(1,1)$ to solve the above problem. In this paper, we do not plan to discuss the conflict in [16]. The main purpose of this paper is to investigate that the simulation curve of original data is consistent with that of the new data by translations. From Table 1, if each original datum is translated by 100 , we see that the new data simulation value of $\operatorname{GGM}(1,1)$ does consist with its corresponding original data simulation curve, but for $\operatorname{GM}(1,1)$, there is not such consistency.

Case 2 (the example for the incidence of Hepatitis B). The following actual value data come from [17] which compared the simulation values of $\operatorname{GM}(1,1)$, improved model [18], Holt-Winters model [18], and GDES and DES models [17], and obtained the idea that the value of MAPE for DES model is minimum. Here, by the aid of the data in [17], we mainly consider the curve consistency. From Table 2, if each original datum is translated by 100000 , as in Case 1, we also see that the new data simulation curve of $\operatorname{GGM}(1,1)$ does consist with its corresponding original data simulation curve, but for $\operatorname{GM}(1,1)$, there is not such consistency.

Case 3 (the example for comparing $\operatorname{DGM}(1,1)$ and $\operatorname{GDGM}(1,1))$. These data come from [4]. The authors [4] compared the simulation values of $\operatorname{GM}(1,1), \operatorname{DGM}(1,1)$, and $\operatorname{OSDGM}(1,1)$ and got that the value of MAPE for $\operatorname{OSDGM}(1,1)$ is minimum. Here, by using the data in [4], we mainly consider the curve consistency. From Table 3, if each original datum is translated by 50 , we see that the new data simulation curve of $\operatorname{GDGM}(1,1)$ does consist with its corresponding original data simulation curve, but for $\operatorname{DGM}(1,1)$, there is not such consistency. 
TABLE 1: Simulation by $\operatorname{GM}(1,1)$ and $\operatorname{GGM}(1,1)$.

\begin{tabular}{|c|c|c|c|c|c|}
\hline Time & Actual value & $\operatorname{GM}(1,1)$ & Actual value +100 & $\operatorname{GM}(1,1)$ & $\operatorname{GGM}(1,1)$ \\
\hline 1 & 6 & 6 & 106 & 106 & 106 \\
\hline 2 & 4 & 4.50 & 104 & 104.15 & 104.50 \\
\hline 3 & 7 & 5.03 & 107 & 104.93 & 105.03 \\
\hline 4 & 5 & 5.62 & 105 & 105.72 & 105.62 \\
\hline 5 & 6 & 6.28 & 106 & 106.51 & 106.28 \\
\hline 6 & 4 & 7.02 & 104 & 107.31 & 107.02 \\
\hline 7 & 10 & 7.85 & 110 & 108.12 & 107.85 \\
\hline 8 & 9 & 8.77 & 109 & 108.93 & 108.77 \\
\hline 9 & 11 & 9.80 & 111 & 109.75 & 109.80 \\
\hline 10 & 10 & 10.96 & 110 & 110.57 & 110.96 \\
\hline MAPE (\%) & & 1.36 & & 0.098 & 0.10 \\
\hline
\end{tabular}

TABLe 2: Simulation by $\operatorname{GM}(1,1)$ and $\operatorname{GGM}(1,1)$.

\begin{tabular}{|c|c|c|c|c|c|}
\hline Month & Actual value & $\operatorname{GM}(1,1)$ & Actual value -100000 & $\operatorname{GM}(1,1)$ & $\operatorname{GGM}(1,1)$ \\
\hline Jan & 162818 & 162818 & 62818 & 62818 & 62818 \\
\hline Feb & 214523 & 200801 & 114523 & 102462 & 100801 \\
\hline Mar & 201184 & 193817 & 101184 & 94260 & 93817 \\
\hline Apr & 155942 & 187077 & 55942 & 86714 & 87077 \\
\hline May & 183216 & 180571 & 83216 & 79773 & 80571 \\
\hline Jun & 165935 & 174291 & 65935 & 73386 & 74291 \\
\hline Jul & 175836 & 168229 & 75836 & 67512 & 68229 \\
\hline Aug & 170885 & 162379 & 70885 & 62107 & 62379 \\
\hline MAPE (\%) & & 0.67 & & 1.39 & 1.43 \\
\hline
\end{tabular}

TABLE 3: Simulation by DGM $(1,1)$ and $\operatorname{GDGM}(1,1)$.

\begin{tabular}{|c|c|c|c|c|}
\hline Actual value & $\operatorname{DGM}(1,1)$ & Actual value -50 & $\operatorname{DGM}(1,1)$ & $\operatorname{GDGM}(1,1)$ \\
\hline 21.1 & 21.1 & -28.9 & -28.9 & -28.9 \\
\hline 26.6 & 22.90 & -23.4 & 18.81 & -27.10 \\
\hline 36.1 & 34.79 & -13.9 & 22.95 & -15.21 \\
\hline 52.3 & 52.85 & 2.3 & 28.01 & 2.85 \\
\hline 80.1 & 80.30 & 30.1 & 34.17 & 30.30 \\
\hline 126.8 & 122.00 & 76.8 & 41.70 & 72.00 \\
\hline MAPE (\%) & 0.48 & & 2.74 & 0.54 \\
\hline
\end{tabular}

\section{Landslide Forecasting in BaZiMen Area}

Landslide is one of universal geological hazards. The people of all countries would like to prevent and control it. The formation of landslide is complicated and related to many factors. Through long-term monitoring on landslide, one finds that forecasting landslide is possible by using statistics method, which offers a useful reference for decision-maker.

Based on the observation data of the landslide involving BaZiMen in three-gorge reservoir area, this paper studies the laws of landslide for a specific period. Let us point out that the aforementioned observation data in terms of the landslide are usually relative to a reference point. Obviously, different reference point has different observation data.

Table 4 is the result of analyzing landslide data between April and September in 2004. The new data simulation curve of $\operatorname{GGM}(1,1)$ coincides with its corresponding original data simulation curve, but for $\operatorname{GM}(1,1)$, there is not such kind of consistency. That is to say, the result of proposed $\operatorname{GGM}(1,1)$ has no relation with reference point but the result of $\operatorname{GM}(1,1)$ is connected with reference point. Thus, $\operatorname{GGM}(1,1)$ improves reliability of simulation forecasting result, which contributes grey system method to landslide forecasting.

\section{Conclusion}

This paper proposes two novel grey models, $\operatorname{GGM}(1,1)$ and $\operatorname{GDGM}(1,1)$. They are the generalization of $\operatorname{GM}(1,1)$ and $\operatorname{DGM}(1,1)$, respectively. Furthermore, we obtain the simulation forecasting curves of two novel grey models which have no relation with translation. The results of practical numerical examples have further demonstrated that the simulation effects of two novel grey models have no relation with reference point. 
TABLE 4: The result of landslide forecasting in BaZiMen area.

\begin{tabular}{|c|c|c|c|c|c|}
\hline YYYYMM & Actual value & $\operatorname{GM}(1,1)$ & Actual value +100 & $\operatorname{GM}(1,1)$ & $\operatorname{GGM}(1,1)$ \\
\hline 2004.04 & 140.7 & 140.7 & 240.7 & 240.7 & 240.7 \\
\hline 2004.05 & 144.4 & 152.72 & 244.4 & 249.79 & 252.72 \\
\hline 2004.06 & 177.8 & 180.43 & 277.8 & 280.41 & 280.43 \\
\hline 2004.07 & 218.5 & 213.17 & 318.5 & 314.78 & 313.17 \\
\hline 2004.08 & 270.4 & 251.85 & 370.4 & 353.36 & 351.85 \\
\hline 2004.09 & 285.2 & 297.54 & 385.2 & 396.68 & 397.54 \\
\hline MAPE & - & 0.69 & - & 0.40 & 0.46 \\
\hline 2004.10 & 300.0 & 351.53 & 400.0 & 445.30 & 451.53 \\
\hline
\end{tabular}

\section{Competing Interests}

The authors declare that there is no conflict of interests regarding the publication of this paper.

\section{Acknowledgments}

This work was supported by Key Laboratory of Geological Hazards on Three Gorges Reservoir Area (China Three Gorges University), Ministry of Education (2015KDZ11); Three Gorges Science Foundation (KJ2013B030, KJ2013B031).

\section{References}

[1] J. L. Deng, "The Control problems of grey systems," Systems and Control Letters, vol. 1, no. 5, pp. 288-294, 1982.

[2] J. L. Deng, The Basis of Grey Theory, Press of Huazhong University of Science \& Technology, Wuhan, China, 2002.

[3] J. Liu, X. Xiao, J. Guo, and S. Mao, "Error and its upper bound estimation between the solutions of $\operatorname{GM}(1,1)$ grey forecasting models," Applied Mathematics and Computation, vol. 246, pp. 648-660, 2014.

[4] N. M. Xie and S. F. Liu, "Discrete grey forecasting model and its optimization," Applied Mathematical Modelling, vol. 33, no. 2, pp. 1173-1186, 2009.

[5] L. Wu, S. Liu, L. Yao, S. Yan, and D. Liu, "Grey system model with the fractional order accumulation," Communications in Nonlinear Science and Numerical Simulation, vol. 18, no. 7, pp. 1775-1785, 2013.

[6] S. H. Mao, M. Y. Gao, X. P. Xiao, and M. Zhu, "A novel fractional grey system model and its application," Applied Mathematical Modelling, vol. 40, no. 7-8, pp. 5063-5076, 2016.

[7] X. Liu, Z. He, and H. Chen, "Partial grey model and it's diagonal transformation," Journal of Grey System, vol. 23, no. 3, pp. 229242, 2011.

[8] Z. S. He, X. Liu, and Y. N. Chen, "Secondary-diagonal mean transformation Partial Grey Model based on matrix series," Simulation Modelling Practice and Theory, vol. 26, pp. 168-184, 2012.

[9] X. Liu and H. Fu, "Volatility forecasting for interbank offered rate using grey extreme learning machine: the case of China," Chaos, Solitons \& Fractals, vol. 89, pp. 249-254, 2016.

[10] Z.-X. Wang and P. Hao, "An improved grey multivariable model for predicting industrial energy consumption in China," Applied Mathematical Modelling, vol. 40, no. 11-12, pp. 5745-5758, 2016.

[11] H.-T. Wang and T.-C. Wang, "Application of the grey LotkaVolterra model to forecast the diffusion and competition analysis of the TV and smartphone industries," Technological Forecasting and Social Change, vol. 106, pp. 37-44, 2016.

[12] L. Z. Li, "Discussion of grey system GM(1,1) model," Mathematics in Practice and Theory, vol. 1, pp. 15-22, 1993.

[13] L. H. Feng, "Discussion of the problem of grey forecast model," Systems Engineering-Theory \& Practice, vol. 12, pp. 125-128, 1997.

[14] X. C. Li, "Widening of suitable limits of grey system GM(1,1) model," Systems Engineering-Theory \& Practice, vol. 1, pp. 97101, 1997.

[15] S. F. Liu, Y. J. Yang, and L. F. Wu, Grey System Theory and Application, Science Press, Beijing, China, 2014.

[16] B. Li, Theory and Application of Economic Forecasting, Economy \& Management Publishing House, Beijing, China, 2005.

[17] L. Wu, S. Liu, and Y. Yang, "Grey double exponential smoothing model and its application on pig price forecasting in China," Applied Soft Computing Journal, vol. 39, pp. 117-123, 2016.

[18] L. Zhang, Y. Zheng, K. Wang, X. Zhang, and Y. Zheng, "An optimized Nash nonlinear grey Bernoulli model based on particle swarm optimization and its application in prediction for the incidence of Hepatitis B in Xinjiang, China," Computers in Biology and Medicine, vol. 49, no. 1, pp. 67-73, 2014. 


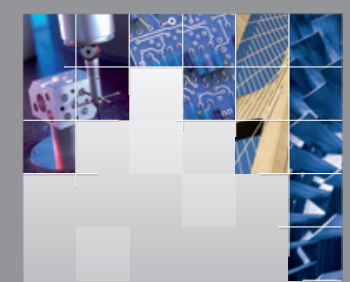

\section{Enfincering}
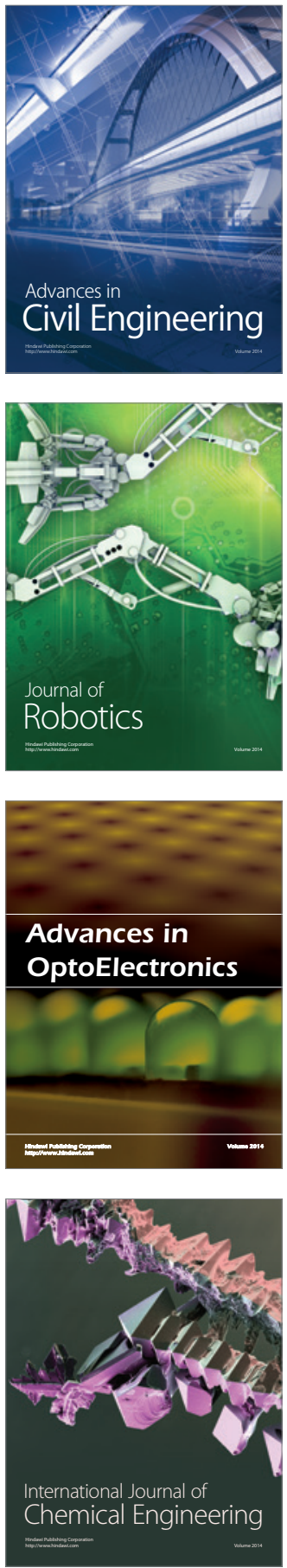

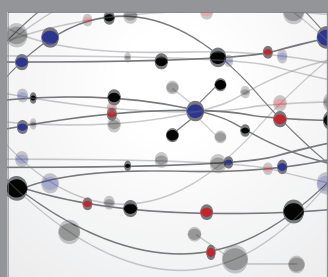

The Scientific World Journal

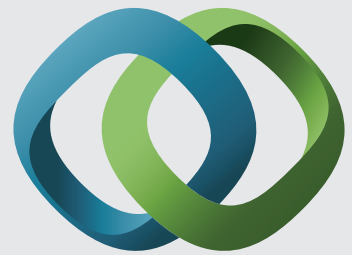

\section{Hindawi}

Submit your manuscripts at

http://www.hindawi.com
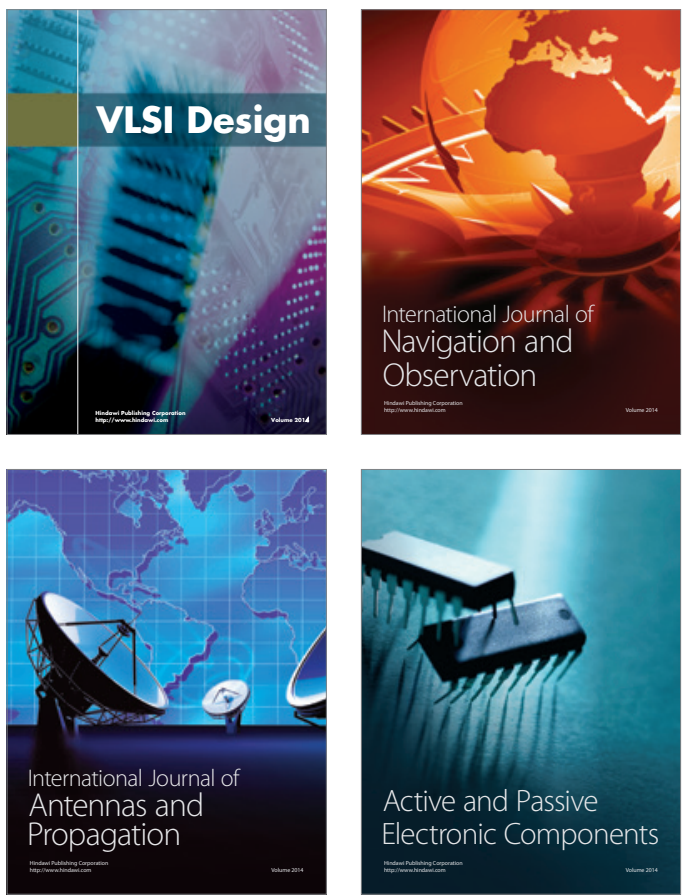
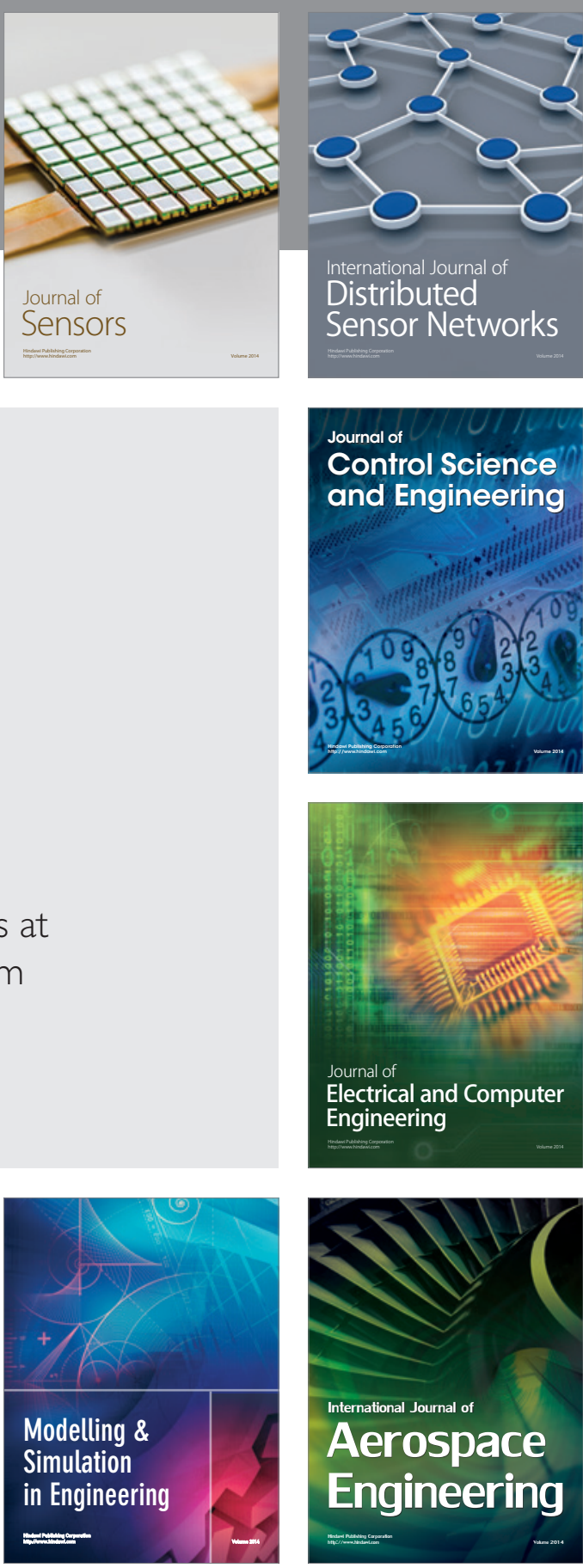

International Journal of

Distributed

Sensor Networks

Journal of

Control Science

and Engineering
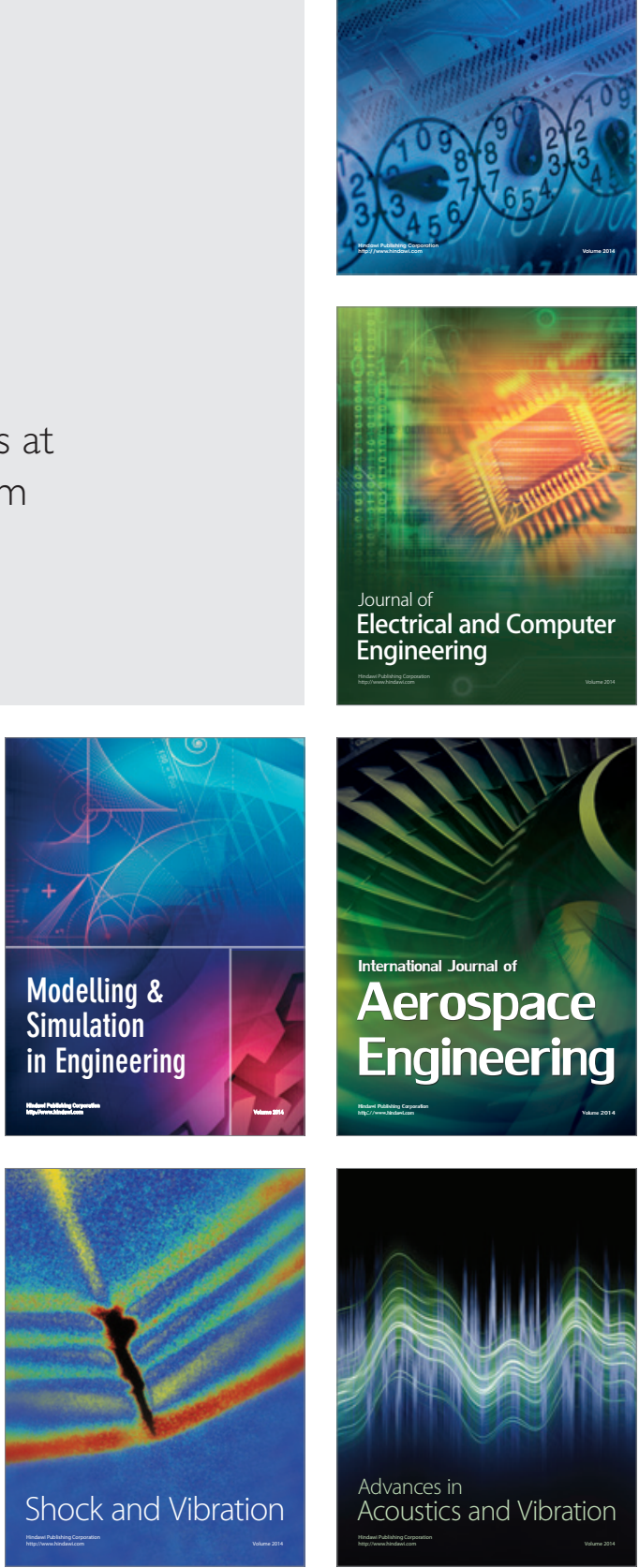